\title{
Theory of pairing symmetry inside the Abrikosov vortex core
}

\author{
Takehito Yokoyama, ${ }^{1}$ Yukio Tanaka, ${ }^{1}$ and Alexander A. Golubov ${ }^{2}$ \\ ${ }^{1}$ Department of Applied Physics, Nagoya University, Nagoya, 464-8603, Japan and CREST, Nagoya, 464-8603, Japan \\ ${ }^{2}$ Faculty of Science and Technology and Mesa + Institute for Nanotechnology, University of Twente, 7500 AE Enschede, The Netherlands
}

(Received 30 May 2008; published 31 July 2008)

\begin{abstract}
We show that the Cooper pair wave function at the center of an Abrikosov vortex with vorticity $m$ has a different parity with respect to frequency from that in the bulk if $m$ is an odd number, while it has the same parity if $m$ is an even number. As a result, in a conventional vortex with $m=1$, the local density of states at the Fermi energy has a maximum (minimum) at the center of the vortex core in an even (odd-) frequency superconductor. We propose a scanning tunneling microscope experiment using a superconducting tip to explore odd-frequency superconductivity.
\end{abstract}

DOI: 10.1103/PhysRevB.78.012508

PACS number(s): 74.20.Rp, 74.50.+r, 74.70.Kn

The study of the mixed state in type-II superconductors has a long history and has revealed a variety of physical phenomena. ${ }^{1}$ In the clean limit, low-energy bound states (the Andreev bound states) are generated in the vortex core due to the spatial structure of the superconducting pair potential. ${ }^{2,3}$ One of the manifestations of the bound states is the enhancement of the zero-energy quasiparticle density of states (DOS) locally in the core, observable as a zero-bias conductance peak by the scanning tunneling microscope (STM). ${ }^{3,4}$ However, despite extensive studies of the vortex core, the issue of pairing symmetry in the core remains unexplored.

Generally, superconducting pairing is classified into an even-frequency or odd-frequency state according to a symmetry with respect to time. Due to the Fermi statistics, evenfrequency superconductors belong to the symmetry class of the spin-singlet even-parity (ESE) or spin-triplet odd-parity (ETO) pairing state, while odd-frequency superconductors belong to the spin-singlet odd-parity (OSO) or spin-triplet even-parity (OTE) pairing state. Although the vortex core state in even-frequency superconductors has been well studied, the vortex core state in odd-frequency superconductors has not been clarified yet.

The possibility of the odd-frequency pairing state in various kinds of uniform systems was discussed in Refs. 5 and 6 , albeit its realization in bulk materials is still controversial. On the other hand, the realization of the odd-frequency pairing state in inhomogeneous even-frequency superconducting systems has recently been proposed. It is established that odd-frequency pairing is induced due to symmetry breaking in such systems. In ferromagnet/superconductor junctions, odd-frequency pairing emerges due to the broken symmetry in a spin space. ${ }^{7}$ It was recently realized that proximityinduced odd-frequency pairing may be generated near normal-metal/superconductor interfaces due to the breakdown of translational symmetry ${ }^{8}$ or in a diffusive normal metal attached to a spin-triplet superconductor. ${ }^{9}$

Since an Abrikosov vortex breaks translational symmetry in a superconductor, one may expect the emergence of a pairing state near the vortex core with a parity different from that in the bulk with respect to frequency.

In this Brief Report, based on the quasiclassical theory of superconductivity, we develop a general theory of pairing symmetry in an Abrikosov vortex core in clean superconductors, including odd-frequency superconductivity. We show that for a vortex with vorticity $m$ in a superconductor, the pairing function of the Cooper pair at the vortex center has the opposite (same) symmetry with respect to frequency to (as) that of the bulk if $m$ is an odd (even) integer. For a conventional vortex with $m=1$, we show that the zero-energy local DOS is enhanced (suppressed) at the center of the vortex core in an even (odd-) frequency superconductor. We further reveal that the OSO $p$-wave pairing is generated at the center of the core of an ESE $s$-wave superconductor. On the other hand, in an OSO $p$-wave superconductor, the ESE $s$-wave pairing state emerges at the center of the vortex core. These results provide a reinterpretation of the Andreev bound states as a manifestation of the odd-frequency pairing. We will also propose an experimental setup to explore oddfrequency superconductivity by probing a local Josephson coupling by STM with a superconducting tip.

The electronic structure of the vortex core in a single Abrikosov vortex in a clean superconductor is described by the quasiclassical Eilenberger equations ${ }^{10,11}$ based on the Riccati parametrization. ${ }^{12}$ Along a trajectory $\boldsymbol{r}\left(x^{\prime}\right)=\boldsymbol{r}_{0}+x^{\prime} \hat{\boldsymbol{v}}_{F}$ with a unit vector $\hat{\boldsymbol{v}}_{F}$ parallel to $\boldsymbol{v}_{F}$, the Eilenberger equations are generally represented in a $4 \times 4$ matrix form. ${ }^{13}$ For a singlet (triplet) superconductor with $\hat{\Delta}=\Delta \sigma_{y}\left(\sigma_{x}\right)$ (where $\sigma_{x}$ and $\sigma_{y}$ are Pauli's matrices in spin space), ${ }^{14}$ these equations are reduced to the set of two decoupled differential equations of the Riccati type for the functions $a\left(x^{\prime}\right)$ and $b\left(x^{\prime}\right)$,

$$
\begin{aligned}
& \hbar v_{F} \partial_{x^{\prime}} a\left(x^{\prime}\right)+\left[2 \epsilon_{n}+\Delta^{\dagger} a\left(x^{\prime}\right)\right] a\left(x^{\prime}\right)-\Delta=0, \\
& \hbar v_{F} \partial_{x^{\prime}} b\left(x^{\prime}\right)-\left[2 \epsilon_{n}+\Delta b\left(x^{\prime}\right)\right] b\left(x^{\prime}\right)+\Delta^{\dagger}=0,
\end{aligned}
$$

where $i \epsilon_{n}$ are the Matsubara frequencies and $\Delta^{\dagger}=(-) \Delta^{*}$ for an even (odd-) frequency superconductor. For a simple case of a cylindrical Fermi surface, the Fermi velocity can be written as $\boldsymbol{v}_{F}=v_{F}\left(\boldsymbol{e}_{1} \cos \theta+\boldsymbol{e}_{2} \sin \theta\right)$.

We choose the following form of the pair potential:

$$
\Delta(\mathbf{r}, \theta, E)=\Delta_{0} \chi(\theta, E) F(r) \exp (\operatorname{im} \varphi),
$$

with $r=\sqrt{x^{2}+y^{2}}$ and $\exp (i \varphi)=(x+i y) / \sqrt{x^{2}+y^{2}}$. Here, $F(r)$ denotes the spatial profile of the gap, $m$ is the vorticity, and $\chi(\theta, E)$ is the symmetry function. Also, we introduce the coherence length $\xi=\hbar v_{F} / \Delta_{0}$; the center of a vortex is situated at $x=y=0$ and $\exp (\operatorname{im} \varphi)$ is the phase factor, which originates 
TABLE I. Pairing symmetry in the vortex state.

\begin{tabular}{lccc}
\hline \hline & Bulk state & Vorticity $m$ & $\begin{array}{c}\text { Symmetry } \\
\text { at the center }\end{array}$ \\
\hline$(1)$ & ESE & Odd & OSO \\
$(2)$ & ESE & Even & ESE \\
$(3)$ & ETO & Odd & OTE \\
$(4)$ & ETO & Even & ETO \\
$(5)$ & OSO & Odd & ESE \\
$(6)$ & OSO & Even & OSO \\
$(7)$ & OTE & Odd & ETO \\
$(8)$ & OTE & Even & OTE \\
\hline \hline
\end{tabular}

from the vortex. To date, various kinds of vortex cores have been studied. ${ }^{15}$ In this Brief Report, we consider axially symmetric cores.

We obtain the pairing function of the Cooper pair (anomalous Green's function) $f$ as $f=-2 a /(1+a b)$. For the calculation of the local DOS normalized by its value in the normal state, the quasiclassical propagator has to be integrated over the angle $\theta$, which defines the direction of the Fermi velocity. The normalized local DOS in terms of functions $a$ and $b$ is given by

$$
N\left(\boldsymbol{r}_{0}, E\right)=\int_{0}^{2 \pi} \frac{d \theta}{2 \pi} \operatorname{Re}\left[\frac{1-a b}{1+a b}\right]_{i \epsilon_{n} \rightarrow E+i \delta},
$$

where $E$ denotes the quasiparticle energy with respect to the Fermi level and $\delta$ is an effective scattering parameter. In numerical calculations throughout this Brief Report, we will fix this value as $\delta=0.1 \Delta_{0}$.

First, we discuss the general property of the symmetry at the vortex center. Vorticity and symmetry of a superconductor with respect to frequency crucially affect the symmetry of the Cooper pair at the core center. Consider a trajectory passing through the center of the vortex. By setting $x^{\prime}=0$ at the vortex center, we get $b\left(x^{\prime}, \epsilon_{n}\right)=-1 / a\left(-x^{\prime},-\epsilon_{n}\right)$ from the Eilenberger equations for an even-frequency superconductor with odd integer $m$ or an odd-frequency superconductor with even integer $m$. Similarly, we obtain $b\left(x^{\prime}, \epsilon_{n}\right)=1 / a$ $\left(-x^{\prime},-\epsilon_{n}\right)$ for an odd-frequency superconductor with odd integer $m$ or even-frequency superconductor with even integer $m$. Thus, at the vortex center $x^{\prime}=0$, we get $f\left(\epsilon_{n}\right)=-f\left(-\epsilon_{n}\right)$ in the former case while $f\left(\epsilon_{n}\right)=f\left(-\epsilon_{n}\right)$ in the latter. Note that spin is conserved in the vortex state considered. Therefore, quite generally, for an odd integer $m$, the induced pairing at the vortex center has a different symmetry with respect to frequency from that in the bulk superconductor. On the other hand, for an even integer $m$, the induced pairing at the vortex center has the same symmetry as that of the bulk. We summarize the pairing symmetry at the vortex center in Table I.

For the conventional $s$-wave case, there have been several studies of the multivortex state with $m \geq 1 .^{16,17}$ It was shown that the zero-energy peak in the DOS only appears for odd number $m$ at the vortex center. ${ }^{17}$ This statement is consistent with our result for the conventional $s$-wave case of $\chi(\theta, E)$ $=1$ because the odd-frequency pairing state is generated only

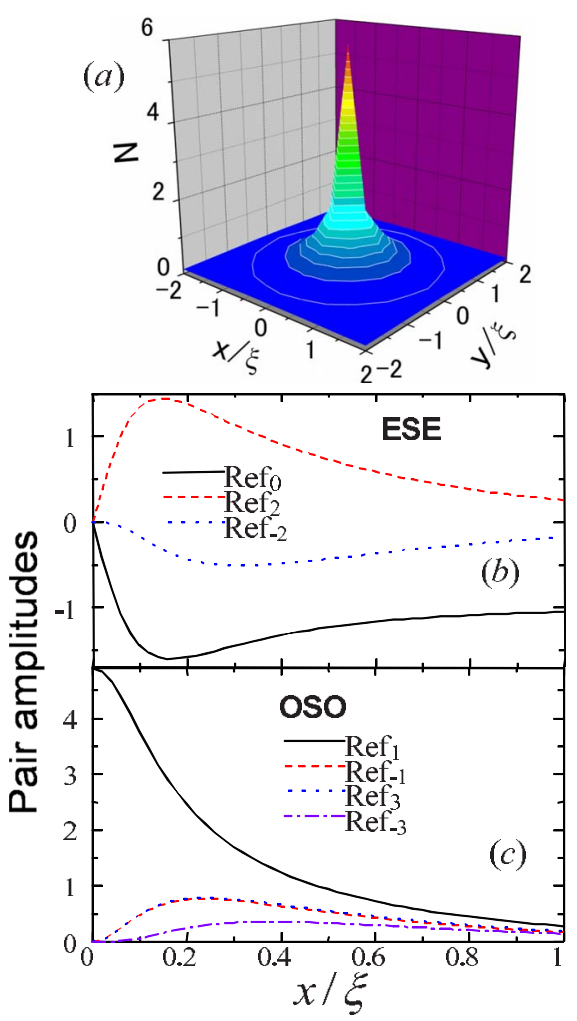

FIG. 1. (Color online) Results for ESE $s$-wave superconductor. (a) Normalized local DOS around the vortex at $E=0$. The center of the vortex is situated at $x=y=0$. Spatial dependencies of (b) ESE and (c) OSO components at $E=0$. Only OSO component $\operatorname{Re} f_{1}$ can survive inside the core (near $x=0$ ).

for odd integer $m$. The relation between the zero-energy peak in DOS and the odd-frequency pairing state will be discussed later.

In general, the most realizable vorticity is $m=1$. Thus, in the following, we will study in detail two typical cases at $m=1$ with ESE $s$-wave and OSO $p_{x}$-wave superconductors where we choose $\chi(\theta, E)=1$ and $\chi(\theta, E)$ $=\left(C \cos \theta E / \Delta_{0}\right) /\left[1+\left(E / \Delta_{0}\right)^{2}\right]$ with $C=0.8$ (Ref. 9), respectively. Also, spatial dependence of the gap is chosen as $F(r)=\tanh (r / \xi)$.

Due to the broken translational symmetry of the system, various pairing states are expected to emerge around the vortex. In order to study possible pairing states, we decompose anomalous Green's function $f$ into various angular momentum components as follows:

$$
f=\sum_{n=0, \pm 1, \pm 2, \ldots,} f_{n} e^{i n \theta} .
$$

Note that all the above pairing components $f_{n}$ are in spin singlet.

Figure 1 shows the results for the ESE $s$-wave superconductor. The local DOS around the vortex at $E=0$ is shown in Fig. 1(a). As is well known, a zero-energy peak appears in the core. ${ }^{3}$ The spatial dependencies of decomposed anomalous Green's function $f$ at $E=0$ are shown in Figs. 1(b) and $1(\mathrm{c})$. Interestingly, only OSO pairing component $\operatorname{Re} f_{1}$ survives at the center of the core. With the increase in the dis- 


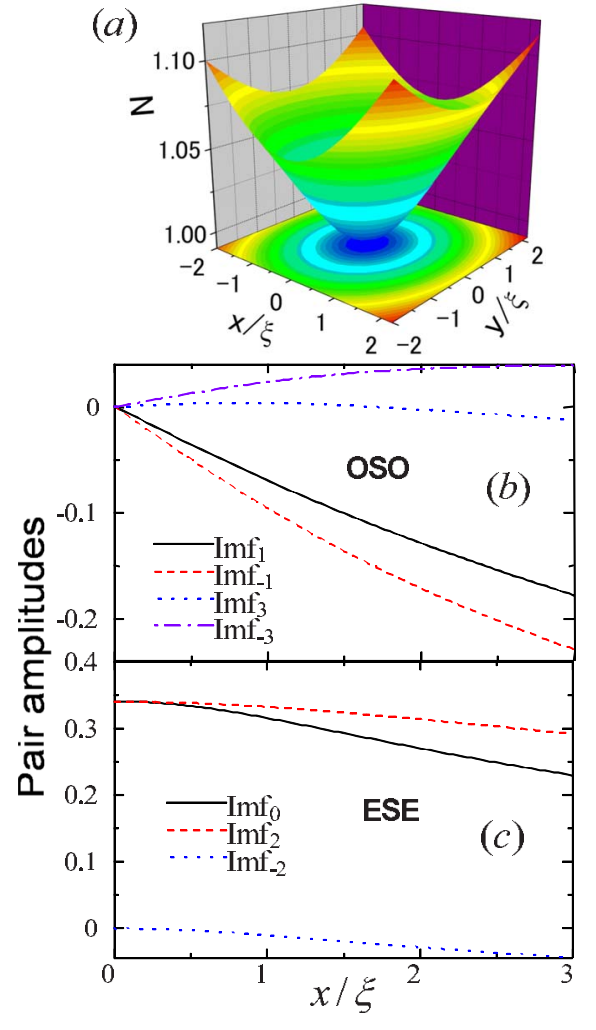

FIG. 2. (Color online) Results for OSO $p_{x}$-wave superconductor. (a) Normalized local DOS around the vortex at $E=0$. The center of the vortex is situated at $x=y=0$. Spatial dependencies of (b) OSO and (c) ESE components at $E=0$. Only ESE components can exist inside the core (near $x=0)$.

tance from the core center, the magnitudes of $f$ decrease rapidly except for $s$-wave one $\left(f_{0}\right)$. Note that other angular momentum components (not shown in this figure) are negligibly small. Thus, we see that the anomalous Green's function at the core center has chiral $p$-wave symmetry.

The enhancement of the local DOS in the presence of odd-frequency pairing can be understood, irrespective of the detailed shape of $\Delta$, by using the normalization condition for the quasiclassical Green's functions $g^{2}+f \bar{f}=1$. Indeed, since in the odd-frequency pairing state, the anomalous Green's function $\bar{f}=-2 b /(1+a b)$ at $E=0$ is given by $\bar{f}(\theta)=-f^{*}(\theta)$ (see Ref. 13) and the local DOS is given by $N(E)=-\operatorname{Re} g$; one can show that generally $N(E=0)>1$ since $g^{2}=1+|f|^{2}$ $>1$. This means that the emergence of the odd-frequency pairing is a physical reason for the zero-energy peak of the local DOS inside the core. The manifestation of the oddfrequency chiral $p$-wave pairing state at the center of the vortex core is also consistent with the experimental fact that the observed zero-bias conductance peak by STM at a vortex center is very sensitive to disorder, ${ }^{18}$ since $p$-wave pairing cannot survive an impurity scattering.

Figure 2 depicts the results for the OSO $p_{x}$-wave superconductor. The local DOS around the vortex at $E=0$ is shown in Fig. 2(a). In dramatic contrast to the result for the ESE $s$ wave, the zero-energy DOS is suppressed at the core. The spatial dependencies of the decomposed anomalous Green's function at $E=0$ are shown in Figs. 2(b) and 2(c). As

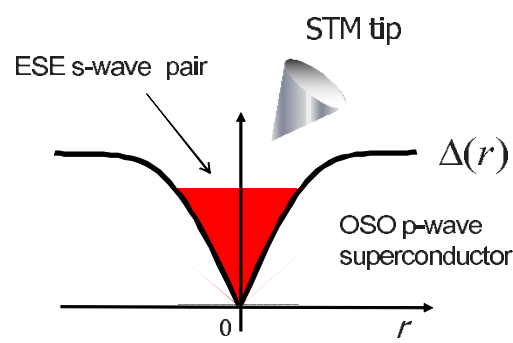

FIG. 3. (Color online) Suggested experimental setup to probe the local Josephson coupling between a superconducting tip and a superconductor with Abrikosov vortex core.

is seen, only ESE pairing components exist at the center of the core. For an even-frequency pairing state, $\bar{f}(\theta)=f^{*}(\theta)$ is satisfied at $E=0$ and hence we get $N(E=0)<1$, which is consistent with Fig. 2(a). By comparing Figs. 1 and 2, it is clear that zero-energy local DOS $N(0)$ has a maximum at the center of the vortex core in an even-frequency superconductor, while it has a minimum at the core center in oddfrequency superconductor. This difference can be detected by STM.

With regards to the candidate for the odd-frequency superconductor, $\mathrm{CeCu}_{2} \mathrm{Si}_{2}$ and $\mathrm{CeRhIn}_{5}$ are possible materials. ${ }^{6,19}$ In these systems, the OSO state with $p$-wave symmetry is considered to be promising. ${ }^{6}$ In the light of the present theory, ESE $s$-wave pairing is expected to appear inside the vortex core. Based on this idea, we propose an experimental setup to verify the existence of odd-frequency pairing in bulk materials by using superconducting STM (Ref. 20), where we use a conventional $s$-wave superconductor as a STM tip ${ }^{21}$ as shown in Fig. 3.

The local Josephson current measured in the STM experiment with the superconducting tip is given by ${ }^{22}$

$$
e I R=\pi T \sum_{\theta, \epsilon_{n}} \operatorname{Im}\left[f^{*}\left(\theta, \epsilon_{n}\right) f_{S}\left(\theta, \epsilon_{n}\right)\right] .
$$

Here, $R$ is the junction resistance, $T$ is the temperature, and $f_{S}$ is the anomalous Green's function in the STM tip. It follows from this expression that a finite Josephson current is allowed only when the superconducting STM has the same symmetry as that in the vortex state. Therefore, a finite $\mathrm{Jo}$ sephson current is allowed at the vortex core only when the pairing symmetry at the core is ESE $s$ wave. Now, we consider a bulk superconductor, which has an ESE $s$-wave symmetry with $m=1$. As seen from Fig. 1, only the OSO state is generated at the core center. Then, the local Josephson coupling is absent at the core while it exists in the bulk. On the other hand, in the OSO $p$-wave superconductor with $m=1$,

TABLE II. Local Josephson coupling with ESE $s$-wave STM tip.

\begin{tabular}{llc}
\hline \hline \multirow{2}{*}{ Bulk state } & \multicolumn{2}{c}{ Local Josephson coupling } \\
& Core & Bulk \\
\hline ESE & No & Yes \\
OSO & Yes & No \\
\hline \hline
\end{tabular}


the ESE $s$-wave state is generated inside the core (see Fig. 2). In this case, the local Josephson coupling exists only at the core. Such a feature is useful to explore and identify oddfrequency pairing in a bulk superconductor. We summarize the possible local Josephson couplings with ESE $s$-wave STM tip in Table II.

In summary, we have developed a general theory of pairing symmetry inside the Abrikosov vortex core in superconductors, including odd-frequency superconductivity. We have found that for a vortex with vorticity $m$ in a superconductor, the anomalous Green's function at the vortex center has the opposite (same) symmetry with respect to frequency to (as) that of the bulk if $m$ is an odd (even) integer. We have also shown that the zero-energy local DOS is enhanced (suppressed) at the center of the vortex core for even (odd-) frequency superconductor. Based on the obtained results, we proposed a STM experiment using a superconducting tip to detect local Josephson coupling in order to explore and identify the odd-frequency superconductor.

T.Y. acknowledges the support by the JSPS. This work was supported by the Ministry of Education, Culture, Sports, Science and Technology of Japan with Grant No. 17071007 and NanoNed Grant No. TSC7029.
${ }^{1}$ G. Blatter, M. V. Feigel'man, V. B. Geshkenbein, A. I. Larkin, and V. M. Vinokur, Rev. Mod. Phys. 66, 1125 (1994); T. Maniv, V. Zhuravlev, I. Vagner, and P. Wyder, ibid. 73, 867 (2001).

${ }^{2}$ C. Caroli, P. G. de Gennes, and J. Matricon, Phys. Lett. 9, 307 (1964); Yu. G. Makhlin and G. E. Volovik, JETP Lett. 62, 737 (1995); A. I. Larkin and Yu. N. Ovchinnikov, Phys. Rev. B 57, 5457 (1998); N. B. Kopnin and G. E. Volovik, Phys. Rev. Lett. 79, 1377 (1997); Phys. Rev. B 57, 8526 (1998); G. E. Volovik, JETP Lett. 70, 609 (1999).

${ }^{3}$ H. F. Hess, R. B. Robinson, R. C. Dynes, J. M. Valles, Jr., and J. V. Waszczak, Phys. Rev. Lett. 62, 214 (1989); F. Gygi and M. Schlüter, Phys. Rev. B 43, 7609 (1991).

${ }^{4} \emptyset$. Fischer, M. Kugler, I. Maggio-Aprile, C. Berthod, and C. Renner, Rev. Mod. Phys. 79, 353 (2007).

${ }^{5}$ V. L. Berezinskii, JETP Lett. 20, 287 (1974); A. Balatsky and E. Abrahams, Phys. Rev. B 45, 13125 (1992); E. Abrahams, A. Balatsky, D. J. Scalapino, and J. R. Schrieffer, ibid. 52, 1271 (1995); P. Coleman, E. Miranda, and A. Tsvelik, Phys. Rev. Lett. 70, 2960 (1993); Phys. Rev. B 49, 8955 (1994); M. Vojta and E. Dagotto, ibid. 59, R713 (1999).

${ }^{6}$ Y. Fuseya, H. Kohno, and K. Miyake, J. Phys. Soc. Jpn. 72, 2914 (2003).

${ }^{7}$ F. S. Bergeret, A. F. Volkov, and K. B. Efetov, Phys. Rev. Lett. 86, 4096 (2001); F. S. Bergeret, A. F. Volkov, and K. B. Efetov, Rev. Mod. Phys. 77, 1321 (2005).

${ }^{8}$ Y. Tanaka, A. A. Golubov, S. Kashiwaya, and M. Ueda, Phys. Rev. Lett. 99, 037005 (2007); M. Eschrig, T. Lofwander, Th. Champel, J. C. Cuevas, and G. Schon, J. Low Temp. Phys. 147, 457 (2007).

${ }^{9}$ Y. Tanaka and A. A. Golubov, Phys. Rev. Lett. 98, 037003 (2007).
${ }^{10}$ G. Eilenberger, Z. Phys. 214, 195 (1968).

${ }^{11}$ A. I. Larkin and Yu. N. Ovchinnikov, Sov. Phys. JETP 28, 1200 (1969).

${ }^{12}$ N. Schopohl and K. Maki, Phys. Rev. B 52, 490 (1995).

${ }^{13}$ M. Eschrig, Phys. Rev. B 61, 9061 (2000).

${ }^{14}$ For triplet pairing, we can choose the $d$-vector parallel to the $\mathrm{Z}$ axis without loss of generality.

${ }^{15}$ M. M. Salomaa and G. E. Volovik, Rev. Mod. Phys. 59, 533 (1987).

${ }^{16}$ G. E. Volovik, JETP Lett. 57, 244 (1993); S. M. M. Virtanen and M. M. Salomaa, Phys. Rev. B 60, 14581 (1999).

${ }^{17}$ Y. Tanaka, H. Takayanagi, and A. Hasegawa, Solid State Commun. 85, 321 (1993); A. S. Mel'nikov and V. M. Vinokur, Nature (London) 415, 60 (2002).

${ }^{18}$ C. Renner, A. D. Kent, P. Niedermann, O. Fischer, and F. Lévy, Phys. Rev. Lett. 67, 1650 (1991).

${ }^{19}$ S. Kawasaki, T. Mito, Y. Kawasaki, G.-q. Zheng, Y. Kitaoka, D. Aoki, Y. Haga, and Y. Onuki, Phys. Rev. Lett. 91, 137001 (2003); G.-q Zheng, N. Yamaguchi, H. Kan, Y. Kitaoka, J. L. Sarrao, P. G. Pagliuso, N. O. Moreno, and J. D. Thompson, Phys. Rev. B 70, 014511 (2004).

${ }^{20}$ J. Smakov, I. Martin, and A. V. Balatsky, Phys. Rev. B 64, 212506 (2001); Phys. Rev. Lett. 88, 037003 (2002); A. V. Balatsky, J. Smakov, and I. Martin, Supercond. Sci. Technol. 15, 446 (2002).

${ }^{21}$ A. Kohen, Th. Proslier, T. Cren, Y. Noat, W. Sacks, H. Berger, and D. Roditchev, Phys. Rev. Lett. 97, 027001 (2006); J. G. Rodrigo, H. Suderow, and S. Vieiraa, Eur. Phys. J. B 40, 483 (2004).

${ }^{22}$ A. V. Zaitsev, Sov. Phys. JETP 59, 1015 (1984). 